\title{
Association of $n$ NOS Gene Polymorphism with Ischemic Stroke in Han Chinese of North China
}

\author{
Yingjie Dai, ${ }^{1}$ Zhiyi He, ${ }^{1}$ Rubo Sui, ${ }^{1}$ Zhilin Jiang, ${ }^{2}$ and Shanshan $\mathrm{Ma}^{3}$ \\ ${ }^{1}$ Department of Neurology, The First Affiliated Hospital of China Medical University, Shenyang 110001, China \\ ${ }^{2}$ Department of Neurology, NO 202 Hospital of People's Liberation Army of China, Shenyang 110001, China \\ ${ }^{3}$ Department of Neurology, Jinqiu Hospital of China Liaoning Province, Shenyang 110001, China
}

Correspondence should be addressed to Zhiyi He; hezhiyil2@yeah.net

Received 30 June 2013; Accepted 30 July 2013

Academic Editors: W. Hall and R. I. Sakakibara

Copyright (C) 2013 Yingjie Dai et al. This is an open access article distributed under the Creative Commons Attribution License, which permits unrestricted use, distribution, and reproduction in any medium, provided the original work is properly cited.

\begin{abstract}
Nitric oxide (NO) is an important messenger molecule and effector molecule. This study aimed to investigate the relation of neuronal nitric oxide synthase (nNOS) gene polymorphism with ischemic stroke in Han Chinese of North China. This was a casecontrol study. A total of 413 patients with ischemic stroke were recruited from Han Chinese of North China. There were 201 males and 212 females. In addition, 477 healthy subjects served as controls including 224 males and 253 females. Multiplex SNaPshot was employed to detect $n N O S$ gene polymorphism (rs2293050, rs2139733, rs7308402, and rs1483757). Results showed that the rs1483757, rs2139733, and rs2293050 genotypes and allele frequencies were comparable between patients and controls. However, ischemic stroke patients had significantly reduced AG genotype and A allele frequency when compared with controls $(P=0.037, P=0.041)$. After adjusting confounding factors (gender, age, smoking, history of drinking, hypertension, and diabetes), AG genotype and A allele were still related to ischemic stroke $(\mathrm{OR}=0.572,95 \% \mathrm{CI}: 0.335-0.978, P=0.041$; $\mathrm{OR}=0.611,95 \% \mathrm{C}$ : 0.378-0.985, and $P=0.041$ ) and both were found to be protective factors. Our results showed that rs7308402 gene polymorphism of nNOS is related to ischemic stroke in Han Chinese of North China.
\end{abstract}

\section{Introduction}

Stroke has a high morbidity, high mortality, high disability, and high recurrence rate and has been a major cause of disability and death worldwide [1]. In China, there are 1500000 to 2000000 new stroke cases every year and $70 \%$ of these are ischemic stroke (IS) cases [2]. It is well known that atherosclerosis, small vessel disease, and arrhythmia are common causes of IS [3], and hypertension, diabetes, and smoking have been found to be risk factors of IS [4]. Increasing evidence shows that IS is caused by multiple factors and as a result of interaction between genetic and environmental factors. It has been confirmed that mutations of MTHFR gene [5], ApoE gene [6], PDE4D gene [7], and $A L O X 5 A P$ [8] are related to the pathogenesis of IS.

$\mathrm{NO}$ is an important messenger molecule and effector molecule. In organisms, NO may serve as a messenger, mediator, or cellular modulator and possesses extensive biological activities [9]. The biosynthesis of NO is regulated by nitric oxide synthase (NOS). According to the biological characteristics and encoding genes, NOS can be classified into neural NOS (nNOS, NOS1), inducible NOS (iNOS, NOS2), and endothelial (NOS, NOS3). eNOS is mainly expressed in the endothelial cells. Under the physiological condition, eNOS derived NO is required for maintenance of circulation system. It can dilate blood vessels, inhibit platelet aggregation, and suppress proliferation of vascular smooth muscle cells exerting antihypertensive and anti-inflammatory effects. Thus, eNOS gene has been regarded as a candidate gene of IS. It has been found that eNOS4a and G894T mutations are the genetic markers of IS [10]. In recent years, animal studies reveal that nNOS derived NO plays a vasoprotective role in the atherosclerosis [11-14]. nNOS derived NO in nonadrenal, noncholinergic autonomic nerves may activate systemic vasculature and together with eNOS derived NO, dilate peripheral, and cerebral blood vessel $[9,15,16]$ and is also involved in the control of blood pressure $[17,18]$. In the cerebral infarction model, NOS1-deficient mice had smaller 
infarct ratio and milder neurological dysfunction when compared with controls, suggesting that NOS1 is detrimental for cerebral infarction $[19,20]$. Thus, nNOS may influence the susceptibility to stroke in a mechanism complementary but different from that of eNOS.

The human NOS1 gene was first identified in the neurons of the brain and thus is also known as neural NOS (nNOS) [21]. It has been confirmed that nNOS is expressed in not only the brain and peripheral nitrogen source nerves but also the kidney, heart, muscular skeletal muscles, vascular smooth muscle cells, and endothelial cells. Human NOS1 (nNOS) gene is mapped to $12 \mathrm{q} 24.2$, and $240 \mathrm{~kb}$ in length. It contains 29 exons and 28 introns. To date, few studies have been conducted to investigate the relationship between $n N O S$ gene polymorphism and pathogenesis of IS. Recently, Manso et al. [22] investigated the NOS1 gene polymorphism in a Portuguese population. Their results showed that the NOS1 gene polymorphism (rs1483757, rs7308402, rs2293050, and rs2139733) was closely related to the pathogenesis of IS. Thus, it is necessary to confirm this finding in different populations. To date, no study has been carried out to explore the relation of NOS gene polymorphism with the pathogenesis of IS in Chinese. This case-control study was undertaken to investigate the association between NOS1 gene polymorphism and IS in Han Chinese of North China. Furthermore, stratification analysis was done according to the gender. Our findings may provide evidence for the prevention of stroke.

\section{Materials and Methods}

2.1. Subjects. Acute ischemic stroke patients $(n=413)$ were recruited from the Department of Neurology of First Affiliated Hospital of China Medical University and NO 202 Hospital of People's Liberation Army of China. There were 201 males and 212 females. Focal neurological deficits were abrupt and acute and continued for more than $24 \mathrm{~h}$. Ischemic stroke was confirmed by brain MRI and/or cranial CT scan. Transient ischemic attack, cerebral embolism, hemorrhagic infarction, cerebral hemorrhage, and subarachnoid hemorrhage were excluded. In addition, cerebral infarction due to cardiogenic events, arteritis, tumors, drugs, trauma, vascular malformations, hematological diseases, or aneurysm was excluded. Concomitant liver and kidney diseases and thyroid disease were not found in these patients. Diagnosis was done by neurologists. Controls $(n=477)$ were the healthy subjects who received routine physical examination in the First Affiliated Hospital of China Medical University. There were 224 males and 253 females. Controls and patients were matched in age and gender. History reviewing, physical examination, and clinical examinations were done in these controls and cerebrovascular diseases; other neurological diseases; kidney/liver diseases, hematological diseases, tumors, peripheral vascular diseases, and autoimmune diseases were excluded from these controls. There was no kinship among all these subjects who were Han Chinese in Liaoning Province, a region of North China. This study was approved by the Ethic Committee of China Medical University and 202 Hospital, and informed consent was obtained from all subjects before study. Questionnaire, physical examination, and laboratory examinations were performed to acquire clinical information of these subjects including age, gender, height, body weight, blood pressure, blood lipid, fasting blood glucose, past history, and history of smoking and drinking (Table 1).

2.2. Genotyping. Venous blood $(3 \mathrm{~mL})$ was collected from each subject and anticoagulated with EDTA. Genomic DNA was extracted with DNA extraction kit (Wizard Genomic DNA purification kit; Promega, USA). UV spectrophotometer was used to determine the concentration and purity of extracted DNA. The genomic DNA was stored at $-20^{\circ} \mathrm{C}$.

Four genetic loci of NOS1 (rs1483757, rs7308402, rs2293050, and rs2139733) were selected according to the gene sequence of NOS1 in dbSNP database of NCBI (http://www. ncbi.nlm.nih.gov/SNP/). SNaPshot multiple Minisequencing technology was used for genotyping [23].

First, genomic DNA underwent amplification by multiplex PCR with the following mixture $(20 \mu \mathrm{L})$ : 1xGC buffer I, $3.0 \mathrm{mM} \mathrm{Mg}^{2+}, 0.3 \mathrm{mM}$ dNTP, $1 \mathrm{U}$ of HotStarTaq polymerase (Qiagen Inc.), $1 \mu \mathrm{L}$ of DNA, and $1 \mu \mathrm{L}$ of multiplex PCR primers. The primers were as follows:

$$
\begin{aligned}
& \text { rs1483757F: } 5^{\prime} \text {-TGCCTCCGACAACTGAGCTGAT- } \\
& 3^{\prime} \\
& \text { rs1483757R: } 5^{\prime} \text {-GCCTGCGTGACAGAGTCAAAT- } \\
& \text { TC-3' } \\
& \text { rs7308402F: 5' -GCAGGCTTATCCCATGGCTCTT- } \\
& 3^{\prime} \\
& \text { rs7308402R: 5'-CCTCTGCTGGGGCATATTTCAA- } \\
& 3^{\prime} \\
& \text { rs2293050F: 5' -ATGGCAGACCTGTGGTGGAG- } \\
& \text { AG-3' } \\
& \text { rs2293050R: 5'-CCCTCCACCGTTTTCCTCACAC- } \\
& 3^{\prime} \\
& \text { rs2139733F: 5' -GAACACCCTGACCTTAGCTGAC- } \\
& 3^{\prime} \\
& \text { rs2139733R: 5' -TTTTGTTGAACCTGGGCCTCTT- } \\
& 3^{\prime} .
\end{aligned}
$$

Amplification was done under the following condition: $95^{\circ} \mathrm{C} 2 \mathrm{~min} ; 11$ cycles of $94^{\circ} \mathrm{C}$ for $20 \mathrm{~s}, 65^{\circ} \mathrm{C}-0.5^{\circ} \mathrm{C} /$ cycle for $40 \mathrm{~s}$ and $72^{\circ} \mathrm{C}$ for $90 \mathrm{~s} ; 24$ cycles of $94^{\circ} \mathrm{C}$ for $20 \mathrm{~s}, 59^{\circ} \mathrm{C}$ for $30 \mathrm{~s}$ and $72^{\circ} \mathrm{C}$ for $90 \mathrm{~s} ; 72^{\circ} \mathrm{C}$ for $2 \mathrm{~min}$. Then, $10 \mu \mathrm{L}$ of PCR products was mixed with $1 \mathrm{U}$ SAP (Promega) and $1 \mathrm{U}$ Exonuclease I (Epicentre) followed by incubation at $37^{\circ} \mathrm{C}$ for $1 \mathrm{~h}$ and $75^{\circ} \mathrm{C}$ for $15 \mathrm{~min}$ for inactivation. The PCR products were purified. SNaPshot multiple single-base extension reaction was done with the following mixture $(10 \mu \mathrm{L}): 5 \mu \mathrm{L}$ of SNaPshot Multiplex Kit (ABI), $2 \mu \mathrm{L}$ of purified products from multiplex PCR, $1 \mu \mathrm{L}$ of primers for extension, and $2 \mu \mathrm{L}$ of ultrapure water. The PCR conditions were $96^{\circ} \mathrm{C}$ for $1 \mathrm{~min}, 28$ cycles of $96^{\circ} \mathrm{C}$ for $10 \mathrm{~s}$, $52^{\circ} \mathrm{C}$ for $5 \mathrm{~s}$, and $60^{\circ} \mathrm{C}$ for $30 \mathrm{~s}$. Then, $10 \mu \mathrm{L}$ of products from extension was mixed with $1 \mathrm{U}$ SAP followed by incubation at $37^{\circ} \mathrm{C}$ for $1 \mathrm{~h}$ and $75^{\circ} \mathrm{C}$ for $15 \mathrm{~min}$ for inactivation. Then, $0.5 \mu \mathrm{L}$ of purified products from extension was mixed with $0.5 \mu \mathrm{L}$ 
TABLE 1: Clinical information of IS patients and controls at baseline.

\begin{tabular}{lccc}
\hline Variables & IS patients $(n=413)$ & Controls $(n=477)$ & $P$ \\
\hline Age & $63.47 \pm 11.57$ & $62.68 \pm 6.32$ & 0.196 \\
Gender $(\mathrm{M} / \mathrm{F})$ & $201 / 212$ & $224 / 253$ & 0.638 \\
BMI $\left(\mathrm{kg} / \mathrm{m}^{2}\right)$ & $24.28 \pm 3.85$ & $24.01 \pm 3.72$ & 0.422 \\
SBP $(\mathrm{mmHg})$ & $143.63 \pm 18.35$ & $132.04 \pm 14.46$ & $<0.001$ \\
DBP $(\mathrm{mmHg})$ & $83.87 \pm 10.32$ & $78.76 \pm 8.82$ & $<0.001$ \\
FBG $(\mathrm{mmol} / \mathrm{L})$ & $6.41 \pm 2.60$ & $5.85 \pm 1.71$ & $<0.001$ \\
TC $(\mathrm{mg} / \mathrm{dL})$ & $5.10 \pm 0.97$ & $4.96 \pm 1.08$ & 0.044 \\
TG $(\mathrm{mmol} / \mathrm{L})$ & $2.02 \pm 3.49$ & $1.63 \pm 1.16$ & $1.33 \pm 0.21$ \\
HDL-C $(\mathrm{mmol} / \mathrm{L})$ & $1.31 \pm 0.32$ & $2.92 \pm 0.62$ & 0.024 \\
LDL-C $(\mathrm{mmol} / \mathrm{L})$ & $2.95 \pm 0.69$ & $174(36.5)$ & 0.149 \\
Hypertension $(n / \%)$ & $261(63.5)$ & $78(16.4)$ & 0.015 \\
Diabetes $(n / \%)$ & $102(24.7)$ & $101(21.2)$ & $<0.001$ \\
Smoking $(n / \%)$ & $172(41.6)$ & $63(13.2)$ & 0.020 \\
Drinking $(n / \%)$ & $122(29.5)$ & & $<0.001$ \\
\hline
\end{tabular}

of Liz120 SIZE STANDARD and $9 \mu \mathrm{L}$ of Hi-Di followed by denaturation at $95^{\circ} \mathrm{C}$ for $5 \mathrm{~min}$. The products were then subjected to sequencing (ABI3130XL). The raw data were analyzed with GeneMapper 4.0 (AppliedBiosystems Co., Ltd., USA) for genotyping.

2.3. Statistical Analysis. Statistical analysis was done with SPSS version 16.0. Data were expressed as mean \pm standard deviation (SD) or percentage. Demographics, risk factors, genotype frequency, and allele frequency were compared with Student's $t$-test or Pearson's $x^{2}$. The correlation of gene polymorphism with IS was analyzed with logistic regression analysis after adjusting traditional risk factors of stroke (such as blood pressure, blood lipid, blood glucose, and history of smoking and drinking). The odds ratios (OR) and 95\% confidence intervals were calculated before and after adjusting confounding factors. Chi-square goodness-of-fit test was used to test whether SNP genotype frequency met the Hardy-Weinberg equilibrium. Online SHEsis software was employed to analyze the linkage disequilibrium and haplotype of 4 SNPs. A value of $P<0.05$ was considered statistically significant.

\section{Results}

Clinical information was collected at baseline from all subjects and compared between patients and controls (Table 1). There were no marked differences in the age, gender, body mass index (BMI), and high-density lipoprotein cholesterol (HDL-C) between the two groups. However, marked differences were noted in the history of hypertension, history of diabetes, history of smoking, history of drinking, systolic blood pressure (SBP), diastolic blood pressure (DBP), fasting blood glucose (FBG), total cholesterol (TC), triglyceride (TG), and low-density lipoprotein cholesterol (LDL-C) between the two groups $(P<0.05)$ (Table 1$)$.

The genotype distribution of rs1483757, rs2139733, rs2293050, and rs7308402 met the Hardy-Weinberg equilibrium $(P>0.05)$. In patients, the $P$ value was $0.635,0.760$,
0.962 , and 0.492 , respectively; in controls, the $P$ value was $0.162,0.111,0.105$, and 0.227 , respectively. This suggests that this population is representative in North China. The genotypes of SNP sites and allele frequency in patients and controls are shown in Table 2. Results showed that there were no marked differences in the genotypes and allele frequency of rs1483757, rs2139733, and rs2293050 between patients and controls $(P>0.05)$. However, the AG genotype frequency and A allele frequency of rs7308402 in IS patients were markedly lower than those in controls $(P=0.037$ and $P=0.041$, resp.). Stratification analysis of females and males revealed that the AG genotype frequency and $A$ allele frequency were different in female patients and female controls $(P=0.001$ and $P=0.002$, resp.). Nevertheless, this difference was not observed between male patients and male controls (Table 3 ). After adjusting confounding factors (such as gender, age, smoking, drinking, hypertension, diabetes, blood lipid, and blood glucose), the AG genotype frequency and A allele frequency were still associated with IS, and both were protective factors $(\mathrm{OR}=0.572,95 \% \mathrm{CI}$ : $0.335-0.978, P=0.041$, and $\mathrm{OR}=0.611,95 \% \mathrm{CI}: 0.378-0.985$, $P=0.041$, resp.) (Table 2). Similar finding was also observed in female patients in stratification analysis $(\mathrm{OR}=0.328$, 95\% CI: $0.153-0.703, P=0.004$, and $\mathrm{OR}=0.347,95 \% \mathrm{CI}$ : 0.174-0.691, $P=0.002$, resp.) (Table 4).

The online SHEsis software was employed to test the pair linkage disequilibrium of 4 sites. Results showed linkage disequilibrium in rs2139733 and $\operatorname{rs} 2293050\left(P_{1}=0.995, P_{2}=\right.$ 0.977). The frequency of potential haplotypes composed of 4 sites (rs1483757, rs2139733, rs2293050, and rs7308402) was also evaluated in patients and controls with SHEsis. Results showed that GATA haplotype frequency in IS patients was dramatically lower than that in controls, suggesting that GATA haplotype is a protective haplotype (OR $=0.593,95 \%$ CI: 0.361-0.977, $P=0.038$ ) (Table 5).

\section{Discussion}

In the present study, 4 SNP sites (rs1483757, rs2139733, rs2293050, and rs7308402) of NOS1 gene were detected in 
TABLE 2: Genotype and allele frequency of 4 sites of NOS1 gene ${ }^{*}$.

\begin{tabular}{|c|c|c|c|c|c|c|}
\hline Genotype & Controls (\%) & Patients (\%) & OR $(95 \% \mathrm{CI})$ & $P$ & Adjusted OR (95\% CI) & $P$ \\
\hline \multicolumn{7}{|l|}{ rs1483757 } \\
\hline AA & $93(22.5)$ & $88(18.4)$ & 1.00 & - & 1.00 & - \\
\hline AG & $211(51.1)$ & $251(52.6)$ & $0.80(0.56-1.12)$ & 0.193 & $0.81(0.56-1.19)$ & 0.284 \\
\hline GG & $109(26.4)$ & $138(28.9)$ & $0.75(0.51-1.10)$ & 0.138 & $0.74(0.48-1.12)$ & 0.737 \\
\hline A allele & $397(48.1)$ & $427(44.8)$ & 1.00 & - & & \\
\hline G allele & $429(51.9)$ & $527(55.2)$ & $1.14(0.95-1.38)$ & 0.163 & & \\
\hline \multicolumn{7}{|l|}{ rs2139733 } \\
\hline $\mathrm{AA}$ & $80(19.4)$ & $83(17.4)$ & 1.00 & - & 1.00 & - \\
\hline AT & $207(50.1)$ & $252(52.8)$ & $0.85(0.60-1.22)$ & 0.381 & $0.87(0.59-1.29)$ & 0.484 \\
\hline TT & $126(30.5)$ & $142(29.8)$ & $0.92(0.62-1.36)$ & 0.677 & $0.92(0.60-1.42)$ & 0.714 \\
\hline A allele & $367(81.3)$ & $418(84.9)$ & 1.00 & - & & \\
\hline T allele & $459(18.7)$ & $536(15.1)$ & $0.99(0.77-1.29)$ & 0.960 & & \\
\hline \multicolumn{7}{|l|}{ rs2293050 } \\
\hline CC & $130(31.5)$ & $143(30.0)$ & 1.00 & - & 1.00 & - \\
\hline CT & $203(49.2)$ & $252(52.8)$ & $0.89(0.66-1.20)$ & 0.431 & $0.86(0.64-1.23)$ & 0.885 \\
\hline TT & 80 (19.4) & $82(17.2)$ & $1.07(0.73-1.58)$ & 0.722 & $1.07(0.70-1.64)$ & 0.764 \\
\hline $\mathrm{C}$ allele & $463(56.1)$ & $538(56.4)$ & 1.00 & - & & \\
\hline T allele & $363(43.9)$ & $416(43.6)$ & $1.05(0.81-1.36)$ & 0.734 & & \\
\hline \multicolumn{7}{|l|}{ rs7308402 } \\
\hline GG & $386(93.5)$ & 427 (89.5) & 1.00 & - & 1.00 & - \\
\hline AG & $27(6.5)$ & $50(10.5)$ & $0.60(0.37-0.97)$ & 0.038 & $0.57(0.34-0.98)$ & 0.041 \\
\hline AA & - & - & - & - & - & - \\
\hline G allele & 799 (96.7) & $904(94.8)$ & 1.00 & - & & \\
\hline A allele & $386(3.3)$ & $434(5.2)$ & $0.61(0.38-0.99)$ & 0.041 & & \\
\hline
\end{tabular}

${ }^{*}$ Adjusting gender, age, hypertension, diabetes, smoking, drinking, serum LDL-C, and blood glucose.

Han Chinese with IS of North China, and their relation with IS was evaluated in this population. Our results showed that the genotypes and allele frequency of rs1483757, rs2139733, and rs2293050 were comparable between IS patients and control, but the AG genotype and A allele frequency of rs7308402 were markedly reduced in IS patients when compared with healthy controls $(P=0.037, P=0.041)$. After adjusting traditional confounding factors (gender, age, hypertension, diabetes, smoking, drinking, and blood lipid), the AG genotype and A allele frequency of rs7308402 were still related to IS (OR $=0.572,95 \% \mathrm{CI}: 0.335-0.978, P=$ 0.041 and $\mathrm{OR}=0.61,95 \% \mathrm{CI}: 0.378-0.985, P=0.041$, resp.). These findings suggest that SNP of rs7308402 may be a genetic marker of female Han patients with IS in North China. Yamaguchi et al. [24] and our previous studies [25, 26] also revealed the difference in some SNPs related to IS between males and females, which was also confirmed in this study. The cause of this difference is still unclear and the difference in sex hormone might be an attributor. Lekontseva et al. [27] investigated the resistant vessels in female animals. Their results showed nNOS mediated dilation of arteries in vitro.

Haplotype analysis may overcome the disadvantage of little information provided by SNP analysis. Haplotype has low recombination rate and high stability. There is evidence showing that haplotype analysis is superior to single SNP analysis, especially when the linkage disequilibrium of SNPs is very weak [28]. Our results showed that GATA (rs1483757rs2139733-rs2293050-rs7308402) was a susceptibility haplotype. The GATA frequency in IS group was markedly lower than that in controls. Subjects with GATA haplotype had a lower risk for IS ( $\mathrm{OR}=0.593,95 \% \mathrm{CI}$ : 0.361-0.977, $P=0.038)$, suggesting that GATA is a protective haplotype.

Studies on nNOS knockout and nNOS overexpression animals significantly improve our understanding of the pathophysiology of nNOS-derived NO [12]. In animals with diet-induced atherosclerosis, ApoE/nNOS knockout mice had more severe atherosclerosis than those with $A p o E$ knockout alone [14]. In addition, nNOS expression is also found in atherosclerotic plaques, suggesting that nNOS plays an important role in the endothelial inflammation [29, 30]. Recently, Chakrabarti et al. [31] found nNOS is involved in the NO production in the endothelial cells at rest, and NO may exert anti-inflammatory effect via reducing proinflammatory cytokines. Thus, $n N O S$ is also regarded as a novel antiatherosclerotic factor. Our results showed that the $n N O S$ gene polymorphism was a protective factor, which was consistent with the antiatherosclerotic property of nNOS. In addition, Nakata et al. [32] found that statins, drugs that can lower cholesterol in hypercholesterolemia patients, could upregulate nNOS expression in human endothelial cells, rat vascular smooth muscle cells, and mouse aorta, suggesting that statins may reduce the risk for stroke in a novel vascular mechanism which is independent of cholesterol-lowering 
TABLE 3: Genotype and allele frequency in males and females.

\begin{tabular}{|c|c|c|c|c|c|c|}
\hline & \multicolumn{3}{|c|}{ Males } & \multicolumn{3}{|c|}{ Females } \\
\hline & Patients (\%) & Controls (\%) & $P$ & Patients (\%) & Controls (\%) & $P$ \\
\hline \multicolumn{7}{|c|}{ rs1483757 } \\
\hline $\mathrm{AA}$ & $48(23.9)$ & $45(20.1)$ & 0.580 & 45 (21.3) & $43(17)$ & 0.328 \\
\hline AG & $99(49.3)$ & $120(53.6)$ & & $112(52.8)$ & $131(51.8)$ & \\
\hline GG & $54(26.9)$ & $59(26.3)$ & & $55(25.9)$ & $79(31.2)$ & \\
\hline A & $195(48.5)$ & $210(46.9)$ & 0.634 & $202(47.6)$ & 217 (42.9) & 0.146 \\
\hline G & $207(51.5)$ & $238(53.1)$ & & $222(52.4)$ & $289(57.1)$ & \\
\hline \multicolumn{7}{|c|}{ rs2139733 } \\
\hline $\mathrm{AA}$ & $41(20.4)$ & $42(18.8)$ & 0.902 & $39(18.4)$ & $41(16.2)$ & 0.591 \\
\hline AT & $98(48.8)$ & $110(49.1)$ & & $109(51.4)$ & $142(56.1)$ & \\
\hline TT & $60(30.8)$ & $72(32.1)$ & & $64(30.2)$ & $70(27.7)$ & \\
\hline A & $180(44.8)$ & $194(43.3)$ & 0.573 & $187(44.1)$ & $224(44.3)$ & 0.960 \\
\hline $\mathrm{T}$ & $222(55.2)$ & $254(56.7)$ & & $237(55.9)$ & $282(55.7)$ & \\
\hline \multicolumn{7}{|c|}{ rs2293050 } \\
\hline $\mathrm{CC}$ & $62(30.8)$ & $73(32.6)$ & 0.842 & $68(32.1)$ & $70(27.7)$ & 0.364 \\
\hline CT & $98(48.8)$ & $110(49.1)$ & & $105(49.5)$ & $142(56.1)$ & \\
\hline TT & $41(20.4)$ & $41(18.3)$ & & $39(18.4)$ & $41(16.2)$ & \\
\hline $\mathrm{C}$ & $222(55.2)$ & $256(57.1)$ & 0.573 & $241(56.8)$ & $282(55.7)$ & 0.734 \\
\hline $\mathrm{T}$ & $180(44.8)$ & $194(42.9)$ & & $183(43.2)$ & $224(44.3)$ & \\
\hline \multicolumn{7}{|c|}{ rs7308402 } \\
\hline GG & $185(92)$ & $210(93.8)$ & 0.492 & $201(94.8)$ & 217 (85.8) & 0.001 \\
\hline AG & $16(8)$ & $14(6.2)$ & & $11(5.2)$ & $38(14.2)$ & \\
\hline $\mathrm{AA}$ & - & - & & - & - & \\
\hline G & $386(96)$ & $434(96.9)$ & 0.499 & $413(97.4)$ & $470(92.9)$ & 0.002 \\
\hline A & $16(4)$ & $14(3.1)$ & & $11(2.6)$ & $36(7.1)$ & \\
\hline
\end{tabular}

TABLE 4: Regression analysis in females ${ }^{*}$.

\begin{tabular}{|c|c|c|c|}
\hline & Adjusted OR & $95 \% \mathrm{CI}$ & $P$ \\
\hline \multicolumn{4}{|c|}{ rs1483757 } \\
\hline $\mathrm{AA}$ & 1 & - & - \\
\hline$A G$ & 0.921 & $(0.533-1.591)$ & 0.768 \\
\hline GG & 0.738 & $(0.405-1.346)$ & 0.322 \\
\hline \multicolumn{4}{|c|}{ rs2139733 } \\
\hline $\mathrm{AA}$ & 1.00 & - & - \\
\hline $\mathrm{AT}$ & 0.775 & $(0.441-1.363)$ & 0.484 \\
\hline $\mathrm{TT}$ & 0.912 & $(0.490-1.696)$ & 0.770 \\
\hline \multicolumn{4}{|c|}{ rs 2293050} \\
\hline $\mathrm{CC}$ & 1.00 & - & - \\
\hline $\mathrm{CT}$ & 0.749 & $(0.466-1.206)$ & 0.234 \\
\hline TT & 1.014 & $(0.547-1.880)$ & 0.964 \\
\hline \multicolumn{4}{|c|}{ rs7308402 } \\
\hline GG & 1.00 & - & - \\
\hline AG & 0.328 & $(0.153-0.703)$ & 0.004 \\
\hline
\end{tabular}

${ }^{*}$ Adjusting gender, age, hypertension, diabetes, smoking, drinking, serum LDL-C, and blood glucose.

effect of nNOS. There is evidence [9] showing that NO in central nervous system is involved in the central regulation of blood pressure and inhibits nNOS activity in the medulla oblongata and hypothalamus resulting in systemic increase in blood pressure. NO from activated peripheral nitrogen source nerves may dilate peripheral blood vessels, reduce peripheral resistance, and then decrease blood pressure [16]. Seddon et al. [15] found that the activated parasympathetic nerves increased NO production in postganglionic fibers of nitrogen source nerves, which increased blood vessels in 
TABLE 5: Haplotype frequency of NOS1 gene in patients and controls.

\begin{tabular}{lccrr}
\hline rs1483757-rs2139733 & Patients (\%) & Controls (\%) & P & OR (95\% CI) \\
-rs2293050-rs7308402 & 9.6 & 9.1 & 0.714 & $1.062(0.771-1.462)$ \\
\hline A A T G & 38.3 & 35.6 & 0.197 & $1.136(0.936-1.378)$ \\
A T C G & 3.0 & 4.9 & 0.038 & $0.593(0.361-0.977)$ \\
G A T A & 31.2 & 29.5 & 0.408 & $1.089(0.889-1.335)$ \\
G A T G & 16.8 & 20.3 & 0.072 & $0.801(0.630-1.020)$ \\
G T C G & & & & \\
\hline
\end{tabular}

Note: Haplotypes with frequency of higher than $3 \%$ are shown.

the brain and increase cerebral blood flow. Taken together, nNOS is directly related to risk factors of stroke such as atherosclerosis and hypertension. Thus, it is necessary to investigate $n N O S$ as a candidate gene of IS.

nNOS protein [21] is composed of PDZ domain, NO synthesis domain, FMN, FAD, and NADH domain. $\mathrm{Ca}^{2+} /$ calmodulin (CaM) mediated nNOS dimerization and depolymerization is switch of nNOS activation, and PDZ domain mediated protein-protein interaction may precisely regulate this switch temporally and spatially. Our results showed stroke related rs7308402 located in intron 2 and thus had no function. Thus, we speculate that there is linkage disequilibrium in the functional site of adjacent extron 2 which encodes PDZ domain, which influences the nNOS activity.

Our results showed rs7308402 was related to IS and served as a protective factor, which was consistent with results in the study of Manso et al. [22]. However, our findings did not reveal the correlation of rs2293050, rs2139733, and rs1483757 with IS, which was not consistent with findings in the study of Manso et al. [22]. Results in different population might be distinct, which might be attributed to the differences in the genetic background and environmental factors, study design, and statistics. Thus, studies with large sample size are required to confirm the relationship between NOS1 gene polymorphism and IS.

Taken together, few studies have been conducted to investigate NOS1 gene polymorphism and IS. Our findings for the first time indicated that AG genotype and A allele at rs7308402 of NOS1 gene may reduce the risk for IS in Han Chinese of North China, especially in females. Thus, both might be protective factors of IS. However, the sample size of our study is still small, the sites of nNOS are also limited, and the NOS1 expression is not detected. Thus, studies with large sample size, more haplotypes, and more examinations are required to functionally confirm the relationship between NOS1 gene and cerebrovascular disease in Han Chinese.

\section{Conflict of Interests}

There is no conflict of interests to disclose.

\section{References}

[1] R. Bonita, S. Mendis, T. Truelsen, J. Bogousslavsky, J. Toole, and F. Yatsu, "The global stroke initiative," Lancet Neurology, vol. 3, no. 7, pp. 391-393, 2004.
[2] M. Liu, B. Wu, W.-Z. Wang, L.-M. Lee, S.-H. Zhang, and L.-Z. Kong, "Stroke in China: epidemiology, prevention, and management strategies," Lancet Neurology, vol. 6, no. 5, pp. 456-464, 2007.

[3] H. P. Adams Jr., B. H. Bendixen, L. J. Kappelle et al., "Classification of subtype of acute ischemic stroke: definitions for use in a multicenter clinical trial," Stroke, vol. 24, no. 1, pp. 35-41, 1993.

[4] L. B. Goldstein, R. Adams, M. J. Alberts et al., "Primary prevention of ischemic stroke. A guideline from the American Heart Association/American Stroke Association Stroke Council: cosponsored by the Atherosclerotic Peripheral Vascular Disease Interdisciplinary Working Group; Cardiovascular Nursing Council; Clinical Cardiology Council; Nutrition, Physical Activity, and Metabolism Council," Stroke, vol. 37, no. 6, pp. 1583-1633, 2006.

[5] J. P. Casas, A. D. Hingorani, L. E. Bautista, and P. Sharma, "Metaanalysis of genetic studies in ischemic stroke: thirty-two genes involving approximately 18000 cases and 58000 controls," Archives of Neurology, vol. 61, no. 11, pp. 1652-1661, 2004.

[6] R. Ariyaratnam, J. P. Casas, J. Whittaker, L. Smeeth, A. D. Hingorani, and P. Sharma, "Genetics of ischaemic stroke among persons of non-European descent: a meta-analysis of eight genes involving 32,500 individuals," PLoS Medicine, vol. 4, no. 4, pp. 728-736, 2007.

[7] J. M. Staton, M. S. Sayer, G. J. Hankey et al., "Association between phosphodiesterase 4D gene and ischaemic stroke," Journal of Neurology, Neurosurgery and Psychiatry, vol. 77, no. 9, pp. 1067-1069, 2006.

[8] A. Helgadottir, A. Manolescu, G. Thorleifsson et al., "The gene encoding 5-lipoxygenase activating protein confers risk of myocardial infarction and stroke," Nature Genetics, vol. 36, no. 3, pp. 233-239, 2004.

[9] U. Förstermann and W. C. Sessa, "Nitric oxide synthases: regulation and function," European Heart Journal, vol. 33, no. 7, pp. 829-837, 2012.

[10] K. Berger, F. Stögbauer, M. Stoll et al., “The glu298asp polymorphism in the nitric oxide synthase 3 gene is associated with the risk of ischemic stroke in two large independent case-control studies," Human Genetics, vol. 121, no. 2, pp. 169-178, 2007.

[11] T. Morishita, M. Tsutsui, H. Shimokawa et al., "Vasculoprotective roles of neuronal nitric oxide synthase," The FASEB Journal, vol. 16, no. 14, pp. 1994-1996, 2002.

[12] V. W. T. Liu and P. L. Huang, "Cardiovascular roles of nitric oxide: a review of insights from nitric oxide synthase gene disrupted mice," Cardiovascular Research, vol. 77, no. 1, pp. 1929, 2008.

[13] M. Tsutsui, "Neuronal nitric oxide synthase as a novel antiatherogenic factor," Journal of atherosclerosis and thrombosis, vol. 11, no. 2, pp. 41-48, 2004. 
[14] P. J. Kuhlencordt, S. Hötten, J. Schödel et al., "Atheroprotective effects of neuronal nitric oxide synthase in apolipoprotein E knockout mice," Arteriosclerosis, Thrombosis, and Vascular Biology, vol. 26, no. 7, pp. 1539-1544, 2006.

[15] M. D. Seddon, P. J. Chowienczyk, S. E. Brett, B. Casadei, and A. M. Shah, "Neuronal nitric oxide synthase regulates basal microvascular tone in humans in vivo," Circulation, vol. 117, no. 15, pp. 1991-1996, 2008.

[16] N. Toda, K. Ayajiki, and T. Okamura, "Cerebral blood flow regulation by nitric oxide: recent advances," Pharmacological Reviews, vol. 61, no. 1, pp. 62-97, 2009.

[17] N. Toda, K. Ayajiki, and T. Okamura, "Control of systemic and pulmonary blood pressure by nitric oxide formed through neuronal nitric oxide synthase," Journal of Hypertension, vol. 27, no. 10, pp. 1929-1940, 2009.

[18] W. T. Talman and D. Nitschke Dragon, "Neuronal nitric oxide mediates cerebral vasodilatation during acute hypertension," Brain Research, vol. 1139, no. 1, pp. 126-132, 2007.

[19] Z. Huang, P. L. Huang, N. Panahian, T. Dalkara, M. C. Fishman, and M. A. Moskowitz, "Effects of cerebral ischemia in mice deficient in neuronal nitric oxide synthase," Science, vol. 265, no. 5180, pp. 1883-1885, 1994.

[20] Z. Huang, P. L. Huang, J. Ma et al., "Enlarged infarcts in endothelial nitric oxide synthase knockout mice are attenuated by nitro-L-arginine," Journal of Cerebral Blood Flow and Metabolism, vol. 16, no. 5, pp. 981-987, 1996.

[21] L. Zhou and D.-Y. Zhu, "Neuronal nitric oxide synthase: structure, subcellular localization, regulation, and clinical implications," Nitric Oxide, vol. 20, no. 4, pp. 223-230, 2009.

[22] H. Manso, T. Krug, J. Sobral et al., "Variants within the nitric oxide synthase 1 gene are associated with stroke susceptibility," Atherosclerosis, vol. 220, no. 2, pp. 443-448, 2012.

[23] M. Bujalkova, K. Zavodna, T. Krivulcik et al., "Multiplex SNaPshot genotyping for detecting loss of heterozygosity in the mismatch-repair genes MLH1 and MSH2 in microsatelliteunstable tumors," Clinical Chemistry, vol. 54, no. 11, pp. 18441854, 2008.

[24] S. Yamaguchi, Y. Yamada, N. Metoki et al., "Genetic risk for atherothrombotic cerebral infarction in individuals stratified by sex or conventional risk factors for atherosclerosis," International Journal of Molecular Medicine, vol. 18, no. 5, pp. 871-883, 2006.

[25] J. Zhao, Z. He, S. Ma, and L. Li, "Association of ALOX15 gene polymorphism with ischemic stroke in Northern Chinese Han population," Journal of Molecular Neuroscience, vol. 47, no. 3, pp. 458-464, 2012.

[26] S. Deng, G. Zhu, F. Liu et al., "CYP4F2 gene V433M polymorphism is associated with ischemic stroke in the male Northern Chinese Han population," Progress in Neuro-Psychopharmacology and Biological Psychiatry, vol. 34, no. 4, pp. 664-668, 2010.

[27] O. Lekontseva, S. Chakrabarti, Y. Jiang, C. C. Cheung, and S. T. Davidge, "Role of neuronal nitric-oxide synthase in estrogeninduced relaxation in rat resistance arteries," Journal of Pharmacology and Experimental Therapeutics, vol. 339, no. 2, pp. 367375, 2011.

[28] R. W. Morris and N. L. Kaplan, "On the advantage of haplotype analysis in the presence of multiple disease susceptibility alleles," Genetic Epidemiology, vol. 23, no. 3, pp. 221-233, 2002.

[29] J. N. Wilcox, R. R. Subramanian, C. L. Sundell et al., "Expression of multiple isoforms of nitric oxide synthase in normal and atherosclerotic vessels," Arteriosclerosis, Thrombosis, and Vascular Biology, vol. 17, no. 11, pp. 2479-2488, 1997.

[30] J. Schödel, P. Padmapriya, A. Marx, P. L. Huang, G. Ertl, and P. J. Kuhlencordt, "Expression of neuronal nitric oxide synthase splice variants in atherosclerotic plaques of apoE knockout mice," Atherosclerosis, vol. 206, no. 2, pp. 383-389, 2009.

[31] S. Chakrabarti, C. K. Chan, Y. Jiang, and S. T. Davidge, "Neuronal nitric oxide synthase regulates endothelial inflammation," Journal of Leukocyte Biology, vol. 91, no. 6, pp. 947-956, 2012.

[32] S. Nakata, M. Tsutsui, H. Shimokawa et al., "Statin treatment upregulates vascular neuronal nitric oxide synthase through Akt/NF- $\kappa$ B pathway," Arteriosclerosis, Thrombosis, and Vascular Biology, vol. 27, no. 1, pp. 92-98, 2007. 


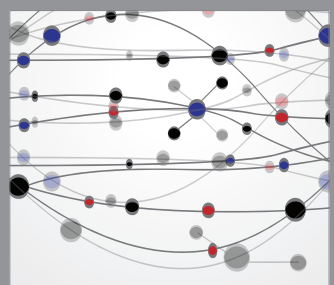

The Scientific World Journal
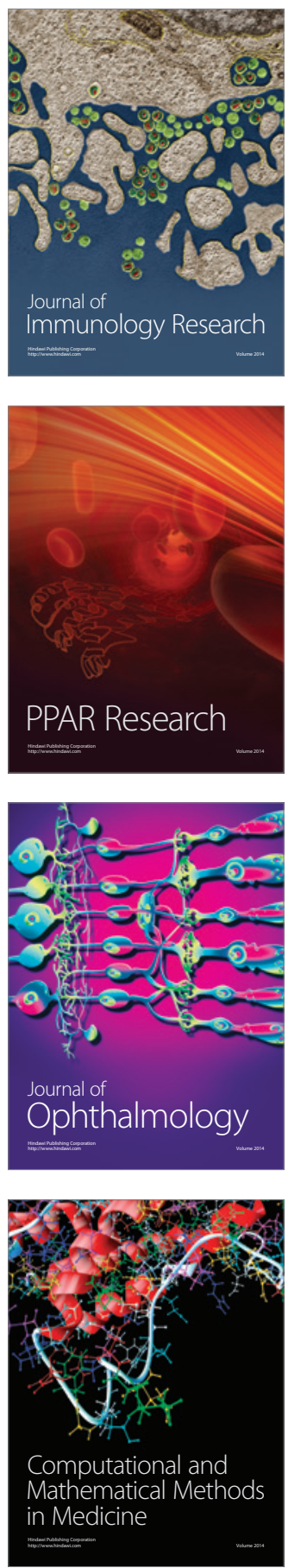

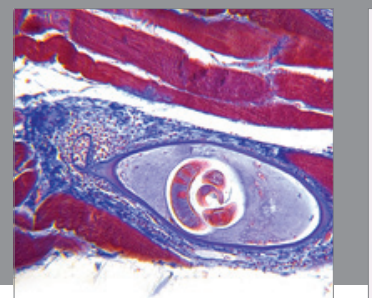

Gastroenterology

Research and Practice
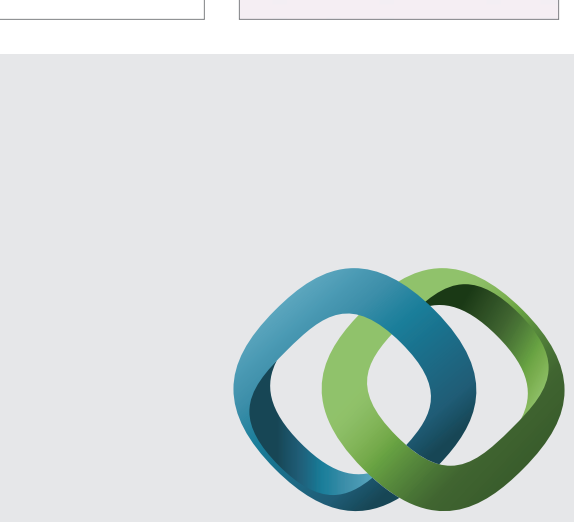

\section{Hindawi}

Submit your manuscripts at

http://www.hindawi.com
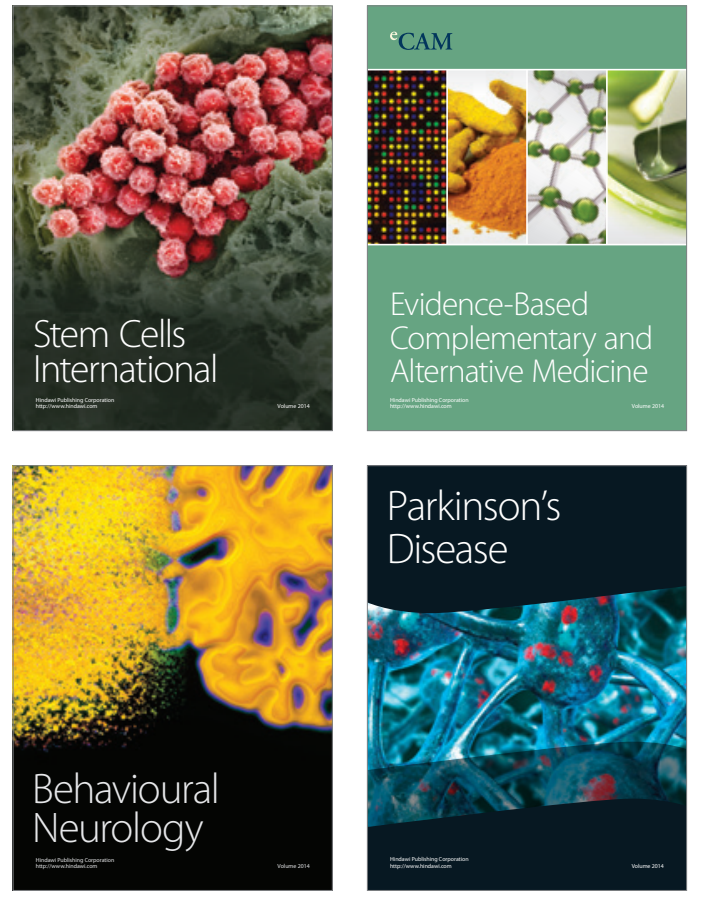
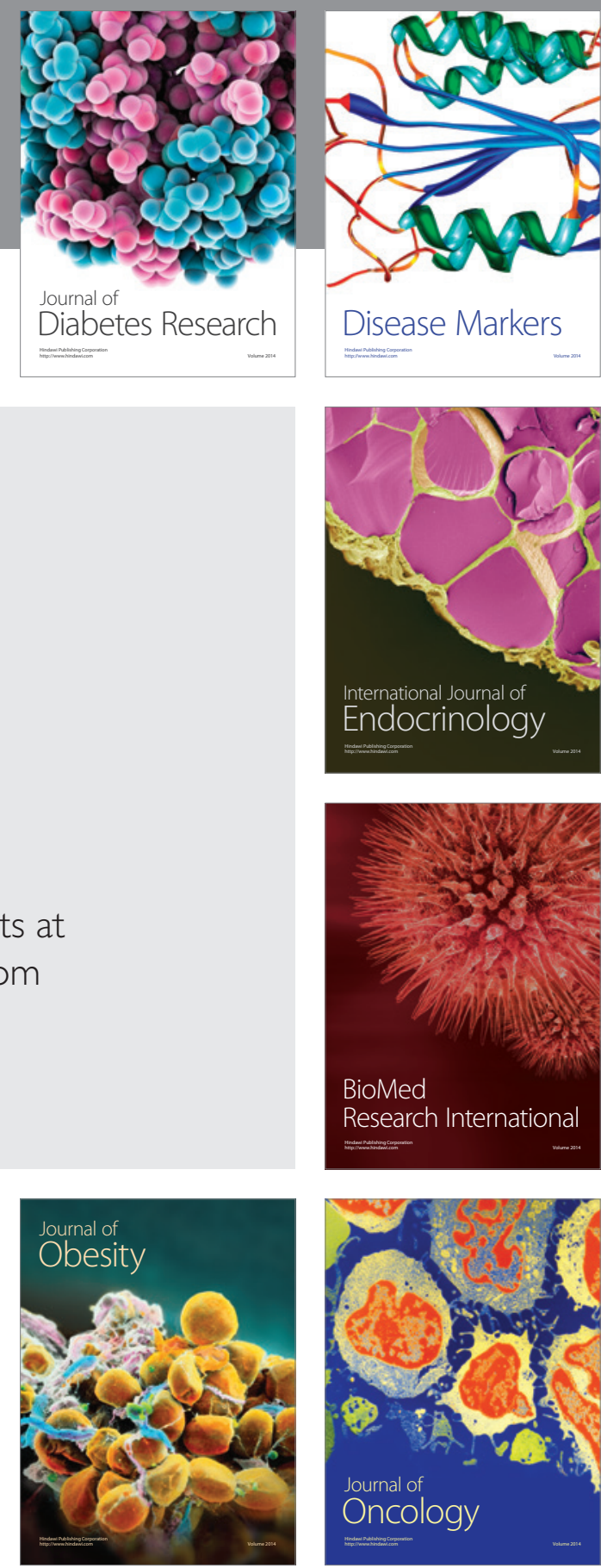

Disease Markers
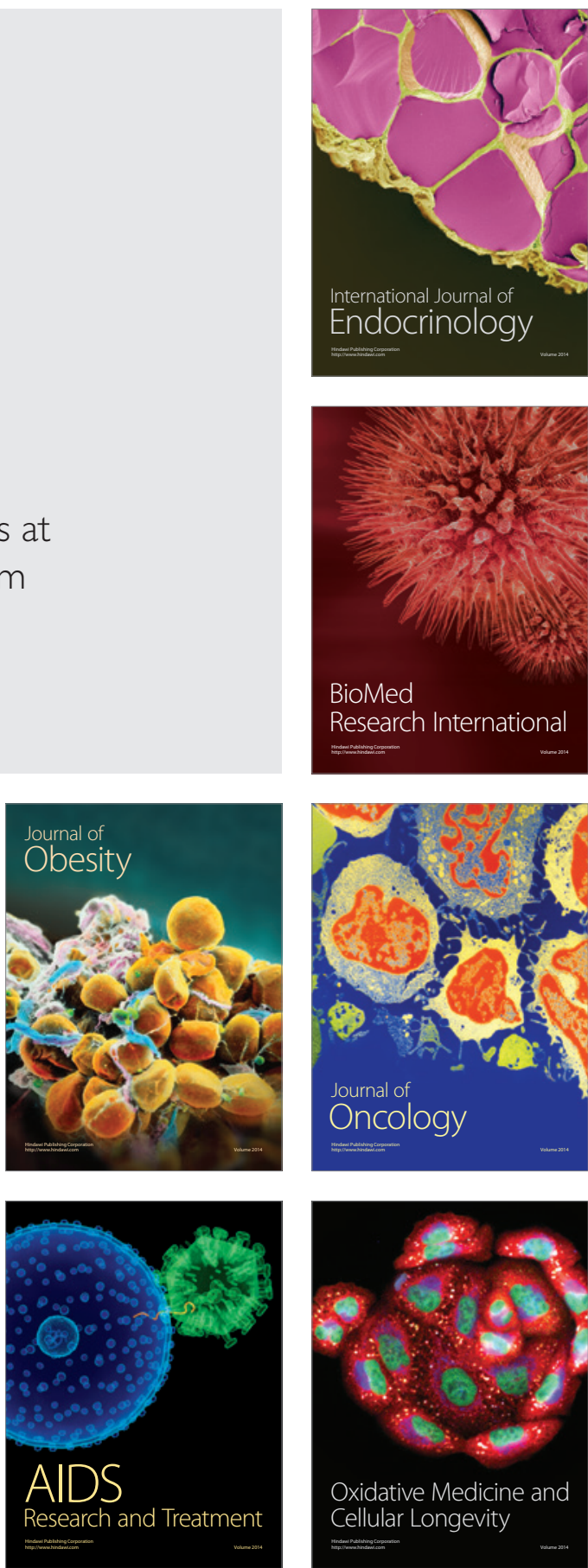